\title{
Estratégias para envolvimento parental em fisioterapia neuropediátrica: uma proposta interdisciplinar
}

\section{Strategies to promote parental involvement in neuropediatric physiotherapy: an interdisciplinary approach}

\author{
Lisandrea Rodrigues Menegasso Gennaro* \\ Universidade Federal de São Carlos - UFSCar, São Carlos, São Paulo, Brasil \\ Elizabeth J oan Barham** \\ Universidade Federal de São Carlos - UFSCar, São Carlos, São Paulo, Brasil
}

\begin{abstract}
RESUMO
Em diversos contextos, amplas evidências mostram que a participação de familiares em intervenções com crianças é de suma importância. No entanto, nos estudos brasileiros na área de fisioterapia, existem poucas informações acerca da eficácia de diferentes estratégias para promover envolvimento parental, ainda que no atendimento a crianças com distúrbios neurológicos, este envolvimento também seja de fundamental importância. Nesse trabalho, identifica-se formas de envolver pais, relatadas nas áreas de Educação Infantil, Educação Especial, Educação Musical e Psicologia (Treinamento de Habilidades Sociais). Discute-se a possibilidade da adaptação das estratégias encontradas para contextos de intervenção fisioterapêutica, para que os profissionais e gestores de instituições de reabilitação ampliem o uso de mecanismos para promover a participação da família, visando maximizar os resultados na reabilitação de crianças. Em pesquisas futuras, seria importante avaliar a viabilidade e eficácia da adoção dessas estratégias, na área de fisioterapia.
\end{abstract}

Palavras-chave: família, fisioterapia, criança, deficiência, reabilitação.

\begin{abstract}
There is ample scientific evidence indicating that the participation of family members in interventions conducted with children is of critical importance. However, little information about the effectiveness of different strategies to increase parental involvement is available in the Brazilian research literature in Physiotherapy, even though family involvement is also of crucial importance in the treatment of children with neurological problems. In this paper, we identify ways of involving parents that are reported in the areas of Early Childhood Education, Special Education, Music Education and Psychology (social skills training). Possibilities for adapting these strategies for use in Physiotherapy settings are discussed, so that professionals and managers of institutions that offer rehabilitation services could broaden their use of mechanisms to promote family participation, maximizing the results of rehabilitation treatment for children. In future studies, it will be important
\end{abstract}


to evaluate the viability and effectiveness of adopting these strategies in Physiotherapy settings.

Key-words: family, physical therapy, disabled, children, rehabilitation.

\section{I ntrodução}

Interdisciplinaridade e integralidade do cuidado são conceitos fundamentais nas esferas de discussão sobre formação em saúde, em contraposição à tendência de fragmentação do conhecimento, resultado do aprofundamento de informações sobre problemas específicos. A literatura científica tem apontado a importância da vinculação da família como colaboradora em contextos de atendimento profissional com a criança, visando à efetividade da intervenção (Dunst \& Dempsey, 2007; Williams \& Aiello, 2004). No entanto, para tratar de demandas complexas e incomuns na população como um todo, existe a tendência de se formar profissionais especializados. Estes profissionais são detentores de informações e técnicas muito pouco conhecidas fora da profissão, com os membros de cada especialidade se dedicando à sua área de atuação. Embora a especialização permita ganhos teóricos e terapêuticos expressivos, o isolamento exagerado das disciplinas tem culminado, muitas vezes, em dificuldades de comunicação com os próprios pacientes e seus familiares, diminuindo a eficiência das práticas de saúde. Por outro lado, interdisciplinaridade se apresenta como resposta à diversidade, à complexidade e à dinâmica do mundo atual. A interdisciplinaridade pode ser compreendida como uma interação e interdependência de diferentes disciplinas em que as potencialidades são exploradas e ampliadas, à medida que se busca o diálogo com outras formas de conhecimento e com outras metodologias, com o objetivo de construir um novo conhecimento (Meireles \& Erdmann, 1999).

No contexto de programas de intervenção com crianças com disfunções neurológicas, não raro, os pais se encontram no papel de consumidores de serviços sobre os quais pouco entendem. Por isso, necessitam das orientações oferecidas pelos profissionais para colaborar adequadamente com o tratamento do filho.

A importância da participação da família, pais ou cuidadores, também tem sido apontada por diversos autores em relação ao tratamento de crianças na área de fisioterapia neuropediátrica (Gração \& Santos, 2008; Foltz, Degangi \& Lewis, 2007; Lima, 2006). Fisioterapia neuropediátrica é a área de fisioterapia que trata de crianças com disfunções neurológicas, como Paralisia Cerebral, Mielomeningocele, Síndrome de Down, lesão medular, dentre outras disfunções.

Assim como outros autores, Foltz et al.(2007) apontam para a necessidade do envolvimento dos pais com os fisioterapeutas para 
maiores ganhos na reabilitação das crianças. O envolvimento dos pais pode auxiliar a criança a se adaptar melhor à fisioterapia, além de fornecer informações sobre os objetivos motores mais importantes para a família, o que favorece o trabalho do terapeuta. Em relação à contribuição dos fisioterapeutas para as famílias, estes podem auxiliar os cuidadores a lidarem com comportamentos inadequados da criança para o tratamento - por exemplo, a preferência em permanecer em posições mais confortáveis, porém inadequadas do ponto de vista da reabilitação; a recusa na utilização de órteses e as manipulações sobre os pais (birras, por exemplo) para evitar exercícios e estratégias incômodas, porém necessárias ao tratamento. Frente a estas situações o fisioterapeuta poderá indicar aos cuidadores como: a) elogiar a criança no momento certo e da forma correta, ou seja, reforçar um comportamento adequado, b) utilizar recursos eficientes para auxiliar na comunicação e c) obter sua colaboração no seguimento às recomendações expressas durante as sessões de fisioterapia. Dessa maneira, as autoras extrapolam a intervenção em fisioterapia para além das patologias e técnicas de intervenção fisioterapêutica, devendo os fisioterapeutas atuar junto à família para que ela estimule as crianças, eduque e lide com as dificuldades inerentes ao processo de reabilitação (Foltz et al., 2007). Sari e Marcon (2008) verificaram a participação de mães de crianças com paralisia cerebral no processo de reabilitação e expectativas em relação ao tratamento. Constataram que a maioria dos familiares não está totalmente esclarecida quanto às limitações associadas à patologia ou então, apresenta-se em processo de aceitação ou negação do diagnóstico. Essas situações constituem indicativos da necessidade de uma atuação mais efetiva por parte dos profissionais junto às mães. As autoras apontam para a necessidade de 0 profissional prover o reforçamento do vínculo pai-mãe e filho; desenvolver a compreensão e a aceitação da patologia; esclarecer os objetivos do tratamento fisioterapêutico; incentivar a participação na escolha e utilização dos métodos de tratamento; conscientizar os pais sobre a importância de sua participação no tratamento e avaliação da evolução; e proporcionar a extensão do tratamento fisioterapêutico em casa, ressaltando seus benefícios. Reforçar, esclarecer, incentivar, conscientizar são grandes classes de comportamento. Como operacionalizar tais comportamentos? Como e quando fazer isso?

A necessidade de o terapeuta realizar algumas destas atividades com - cuidador é tão fundamental que nas Diretrizes Curriculares Nacionais para o curso de Fisioterapia está prescrito que é dever do profissional prestar esclarecimentos, dirimir dúvidas e orientar os indivíduos e seus familiares sobre o processo terapêutico (Resolução CNE/CSE 4/2002). Todavia, não é tratado, especificamente, do envolvimento parental mais próximo na intervenção com crianças. Também não foram encontrados documentos, indicadores ou 
recomendações para o profissional da área de fisioterapia se comportar em relação à família com o objetivo de obter sua colaboração e envolvimento com a reabilitação da criança com condições crônicas de saúde.

A pouca visibilidade de estratégias para garantir o envolvimento parental se reflete na escassez de pesquisas que descrevem e avaliam a eficácia de diferentes táticas para promoção do envolvimento parental na reabilitação de crianças na literatura brasileira na área de fisioterapia, conforme indica estudo de Menegasso e Barham (2011). As autoras realizaram um estudo de revisão em periódicos de fisioterapia e constataram que, em grande parte das pesquisas, a participação dos pais consiste em oferecer ao fisioterapeuta informações para avaliação da criança, delineamento do tratamento e escolha de técnicas de intervenção (por exemplo, os estudos de Amorim et al., 2009; Vasconcelos, Moura, Campos, Lindquist \& Guerra 2009; Prado, Magalhães \& Wilson, 2009; Santos et al., 2009; Silva \& Daltrário, 2008). O levantamento identificou, em menor quantidade, outras pesquisas que evidenciam a influência das práticas maternas no desenvolvimento motor das crianças e indicam a necessidade de intervenção junto às mães (Gração \& Santos, 2008; Silva, Santos \& Gonçalves, 2006).

Entre as poucas estratégias já avaliadas na literatura de fisioterapia para promoção do envolvimento parental, autores como Cruz, Ide, Tanaka e Caromano (2008) e Martinez, Joaquim, Oliveira e Santos (2007) verificaram a eficácia do uso de manuais para pais com o objetivo de ensinar práticas de massagem e estimulação da criança, respectivamente (Menegasso \& Barham, 2011).

Lima (2006) atenta para necessidades de formação para que o fisioterapeuta lance seu olhar tanto para a criança como para sua mãe. Aponta, ainda, para a importância de prática interdisciplinar e estudos interdisciplinares para contribuir com melhores níveis de interação mãe-filho e mãe-profissional de saúde, adesão e participação das mães no tratamento de seus filhos visando melhor desenvolvimento neuropsicomotor e qualidade de vida das crianças com deficiência.

Ainda que na área de fisioterapia a explicitação de estratégias para a interação profissional-familiar seja rara, as áreas de conhecimento Educação Infantil, Educação Especial e Educação Musical e Habilidades Sociais são ricas em oferecer e documentar táticas para envolvimento familiar em contextos de intervenção com crianças. Considerando-se a importância do envolvimento parental na reabilitação dos filhos e diante a escassez de estratégias explícitas para promoção desse envolvimento na literatura brasileira na área de fisioterapia, percebe-se que há a necessidade de se adotar uma perspectiva interdisciplinar. Dessa forma, esse estudo tem como objetivo identificar estratégias para envolvimento parental em outros 
contextos de intervenção com os filhos, de modo a suprir essa lacuna. As áreas do conhecimento: Educação Infantil, Educação Especial, Educação Musical e Habilidades Sociais foram selecionadas porque oferecem estratégias para envolvimento parental em outros contextos de intervenção com crianças.

\section{Estratégias para envolvimento parental em contextos de educação infantil}

No Brasil, o Ministério da Educação disponibiliza publicações que permitem aos dirigentes de instituições de educação infantil reconhecer e adotar estratégias para participação da família na educação dos filhos. Os "Critérios para um atendimento em creches que respeite os direitos fundamentais das crianças" (Campos \& Rosemberg, 2009) são produto de anos de pesquisa e trata do funcionamento de creches e políticas. É redigido no formato de afirmações positivas, com o propósito de assegurar o compromisso de um atendimento de qualidade em creche voltado para as necessidades fundamentais da criança, incorporando a família nesse processo. Esse material pode ser adotado como um roteiro para implantação e avaliação de indicadores de qualidade, bem como um termo de responsabilidade. Entre os indicadores relacionados ao envolvimento familiar com a creche estão: a existência do diálogo aberto e contínuo para ajudar a responder às necessidades individuais da criança; o estímulo para participar ativamente de eventos e atividades na creche; orientação da família sobre a importância das brincadeiras para o desenvolvimento infantil; oferecimento de informações sobre o desenvolvimento das crianças; existência de espaços acolhedores para receber e conversar com as famílias.

Na mesma direção, o documento "Indicadores de Qualidade na Educação Infantil" (Ministério da Educação [MEC], 2009) foi construído com o objetivo de auxiliar as equipes que atuam na educação infantil, juntamente com famílias e pessoas da comunidade, a participar de processos de autoavaliação da qualidade de creches e pré-escolas. Entre os indicadores de qualidade são avaliados quesitos como: garantia do direito das famílias de acompanhar as vivências e produções das crianças; atualização periódica da proposta pedagógica com a participação das professoras, demais profissionais e famílias; existência de espaços especialmente planejados para recepção e acolhimento dos familiares; reuniões periódicas com os familiares para apresentar planejamentos, discutir e avaliar as vivências e produções das crianças.

Pesquisadores brasileiros têm analisado uma escala americana de avaliação da qualidade de ambientes de educação infantil, na qual 
são apontados caminhos para aumentar a participação dos pais, uma vez que níveis de maior inserção dos pais estão associados à qualidade na educação infantil (Souza \& Campos-de-Carvalho, 2005; Menegasso-Gennaro \& Gil, 2012; Campos, Esposito, Bhering, Gimenes, \& Abuchaim, 2011), a Infant/Toddler Environment Rating Scale - Revised Edition (Harms, Cryer, \& Clifford, 2003). O instrumento permite avaliar e promover a qualidade do atendimento à criança em ambientes coletivos de educação infantil. É composto por 39 itens, distribuídos em sete subescalas, entre elas a subescala "Pais e equipe de trabalho" que trata da oportunidade de envolvimento dos pais.

A escala permite avaliação por meio de escores que variam de "inadequado" a "excelente" e, em sendo "excelente", devem ser observados as seguintes características em relação ao envolvimento dos pais: conscientização sobre a filosofia e abordagens do programa, existência de manual de pais; políticas de disciplina; descrição das atividades; reunião de orientação dos pais; bastante compartilhamento de informações entre pais e a equipe educacional sobre a criança (por exemplo, recados informais frequentes, folhetos informativos, disponibilidade de informações sobre saúde, segurança e desenvolvimento infantil); utilização de uma variedade de alternativas para incentivar o envolvimento da família (por exemplo, participação em refeições e festas). A escala também prevê a avaliação anual do programa pelos pais, por meio de questionário e reuniões para avaliação em grupo; encaminhamento dos pais para outros profissionais quando necessário e decisão conjunta sobre o programa com a equipe da direção (Harms et al., 2003).

De forma muito similar ao que foi recomendado na área de Educação Infantil, são encontradas recomendações na área de Educação Especial.

\section{Envolvimento parental em contextos de Educação Especial}

Araújo (2004), tratando do envolvimento de famílias e profissionais de educação especial para o atendimento de crianças, aponta para a importância do envolvimento familiar e a potencialização do tratamento quando os pais agem como mediadores desse processo. Argumenta que profissionais da área de saúde e educação podem oferecer suporte informacional e apoio social à família. Entre as possibilidades de estratégias para promover o envolvimento da família, indica: organização de reuniões periódicas; discussão passo a passo sobre as dificuldades da pessoa com necessidades especiais; tomar decisões conjuntas sobre os modos de intervir com a criança; acompanhamento e fornecimento de feedback e avaliação conjunta do processo. A autora atuou com grupos de apoio para mães de 
crianças com deficiência, onde observou benefícios para a criança com deficiência, para a família, bem como para os profissionais envolvidos e comunidade. Entre os benefícios para a família no contexto de intervenção em educação e saúde, estão: a) a possibilidade de receber informações esclarecedoras sobre a importância da família como ambiente inclusivo, b) reconhecer os pontos fortes e fracos do membro familiar com deficiência e aprender a programar atividades para ele, c) aprender a envolver outros membros da família, d) aprender a ensinar novas habilidades no próprio lar. Em relação à pessoa com deficiência, esta pode se beneficiar porque tem maior probabilidade de participação na família e na vida em comunidade. Já para os profissionais de educação e saúde, estes podem atingir melhor seus objetivos porque terão mais informações sobre as habilidades e dificuldades da família, conseguindo planejar atividades que possam realmente ser conduzidas dentro do lar e assim aumentar a efetividade do tratamento. Para Araújo (2004), a aliança família-profissional oferece aos integrantes da tríade oportunidade para trabalhar cooperativamente na resolução de problemas mútuos de maneira não ameaçadora ou defensiva, especialmente se o profissional estiver atento a: conhecer suas próprias concepções, atitudes e valores, conhecer o sistema familiar considerando e valorizando as diferenças culturais, reconhecer os limites da condição socioeconômica e, por fim, desenvolver respeito e confiança mútuos.

Convergindo para as mesmas conclusões, Dessen e Pereira-Silva (2004) afirmam que o envolvimento de diversos membros da família em programas de intervenção é o meio mais eficaz para atingir os objetivos compartilhados no atendimento à criança. Nos Programas de Estimulação Precoce, por exemplo, não só a criança, mas também toda a família, pode se beneficiar com a atenção profissional, especialmente em se tratando de intervenções em serviço social, psicologia ou outra especialidade que possa ampliar as condições de desenvolvimento da família, principalmente em relação aos recursos psicológicos e à qualidade das interações familiares. Um programa de intervenção deve, portanto, envolver o maior número possível de membros familiares.

Williams e Aiello (2004) lançam luz ao conceito de empoderamento dos pais, que salienta a importância da parceria do profissional com o familiar no desenvolvimento social da criança com necessidades especiais, porque sua atuação é de agente de transformação social. 0 empoderamento é o processo pelo qual as famílias conquistam as condições para adquirir conhecimentos, habilidades e recursos para que tenham melhor controle de sua situação e melhoraria da sua qualidade de vida.

Embora ainda não exista uma medida específica de empoderamento, a escala Family Environment Scale - FES (Koren, De Chillo, \& 
Friesen, 1992) investiga parte dos comportamentos que reflete a capacidade do respondente de experimentar, avaliar e decidir sobre o uso de serviços e apoios sociais disponíveis. Essa escala é composta por 34 itens para avaliar: a) como a família lida com situações cotidianas no contexto imediato, b) como a família lida com os profissionais e as instituições que prestam serviço a seus filhos e c) 0 que a família faz para exercer seus direitos como cidadãos. Em relação ao segundo aspecto, lidar com profissionais e instituições, consta itens que remetem à consciência que os pais têm em relação: a) ao seu direito de aprovar ou não os serviços que o filho recebe, b) à capacidade para contribuir com os serviços para crianças, c) à necessidade de ter sua opinião levada em conta pelos profissionais, d) à compreensão sobre a organização dos serviços oferecidos, e) ao poder de decisão quanto à seleção, adaptação e aceite dos serviços oferecidos, f) à capacidade de atuar em conjunto com profissionais e instituições para decidir sobre os serviços a serem usados no tratamento de seu filho, g) à possibilidade de oferecer idéias e sugestões para melhoria dos serviços prestados (Silva, 2007).

Aponta-se para a importância do empoderamento dos cuidadores pelos fisioterapeutas para melhorar a compreensão e aproveitamento dos serviços disponibilizados, e, consequentemente, a ampliação envolvimento parental com o tratamento da criança.

A fisioterapia em crianças com disfunções neurológicas também engloba ensinar a criança a realizar movimentos específicos com seu corpo, tal como ocorre na Educação Musical, que será descrito a seguir.

\section{Envolvimento parental na Educação Musical}

Sob a ótica dos benefícios de uma atuação colaborativa do profissional e a família de uma criança que recebe um serviço, qualquer professor deveria apreciar o envolvimento dos pais na educação dos filhos. No entanto, no contexto da educação musical, embora esta visão seja pouco comum, o Método Suzuki ${ }^{\mathrm{Tm}}$ aborda este envolvimento explicitamente. Os fundadores da associação de professores Suzuki determinaram que o professor necessita estabelecer uma espécie de sociedade com os pais, e estes devem desempenhar um papel ativo na formação musical da criança. Tal método de educação musical foi desenvolvido por Sinichi Suzuki no século passado e baseia-se em alguns princípios que o torna diferente da educação musical tradicional (Barber, 1991).

A filosofia Suzuki é baseada no preceito de que as crianças são educadas por meio do ambiente construído para elas e, nesse paradigma, ressalta a importância do envolvimento dos pais na educação musical da criança. O pai (ou a mãe) deve participar de 
cada aula do filho, preparando-o para ser o professor da criança em casa, até a próxima aula com o professor. Assim, deve tomar notas das informações relevantes e tirar dúvidas de maneira a poder conduzir o treinamento do filho ao longo da semana. Algumas vezes, ele mesmo deve aprender a tocar as peças no instrumento, primeiramente ou paralelamente à aprendizagem do filho, para poder auxiliar com maior propriedade. Esse engajamento requer organização e compromisso dos pais, que precisam gerenciar seu tempo e energia para desempenharem o papel de professores de música no contexto doméstico (Barber, 1991).

Além da inserção do pai na sala de aula, o método tem produzido literatura voltada a potencializar o papel do pai durante o exercício em casa, promovendo motivação, conscientização do papel do ambiente e da família no desenvolvimento da criança, fornecendo recomendações e dicas (Sprunger, 2005). Exemplos de recomendações vão desde estacionar o carro de maneira correta em frente a escola e desligar o telefone celular durante a aula à comparecer a workshops e observar a aula de outros alunos. Outras recomendações do método são: trazer material para a aula, manter atitude positiva, identificar pontos principais de cada lição, aprender aos poucos, isolar os pontos da lição que são difíceis de executar, fazer com que a criança execute os pontos difíceis repetidamente para poder aprender coisas novas, fazer perguntas ao professor e comparecer a todos os recitais (para verificar resultados futuros possíveis e demonstrar a alunos menos avançados quais os resultados do estudo regular e comprometido).

Assim, a literatura sobre educação musical de crianças tem nos pais um público alvo. Por exemplo, Sprunger (2005), autor com formação na perspectiva Suzuki e formação também no campo da Psicologia, escreveu Helping Parents Practice: I deas for making it easier. O livro conta com 74 lições para os pais a fim de capacitá-los para promover a aprendizagem do filho para tocar instrumentos musicais.

Uma forte recomendação que permeia o livro é o incentivo ao ensaio diário e a repetição na execução da música. Enquanto o ensaio diário é propiciado pelo estabelecimento de rotinas, o exercício de repetição precisa de estratégias melhor elaboradas. Assim, no livro, o autor explica a importância da repetição diária de uma música nova pela criança para que domine as habilidades específicas, sendo que os pais precisam entender que a única maneira de tornar o processo de aprendizagem mais fácil seria com base no domínio cumulativo das habilidades técnicas envolvidas em cada peça do repertório, pela criança. Adicionalmente, apresenta diversas estratégias para que os pais tornem a repetição mais atraente para as crianças, como por exemplo, utilização de jogos ou atividades nas quais, a cada execução adequada do movimento, é criada a oportunidade da 
criança realizar uma atividade lúdica concreta que simbolize seu percurso durante o exercício.

Tais estratégias apresentadas na literatura sobre Educação Musical oferecem fortes exemplos e recursos explícitos para o engajamento dos pais com os profissionais, com os filhos e no processo de educação musical das crianças.

\section{Envolvimento parental no desenvolvimento de Habilidades Sociais por parte dos filhos}

Dentro do campo do desenvolvimento infantil existem subáreas de pesquisa que apontam, não apenas na teoria, mas com dados empíricos, a influência dos pais no desenvolvimento saudável dos filhos. No campo das Habilidades Sociais, por exemplo, Del Prette e Del Prette (2005) notam que a aprendizagem de comportamentos sociais e de normas de convivência social inicia-se na infância. As condições ambientais oferecidas no contexto familiar influenciam principalmente nas relações interpessoais da criança, na medida em que promovem 0 processo de aprendizagem por observação, modelação, instrução e consequenciação. O contexto familiar constitui a base da estimulação de padrões de desempenho e competência social. No entanto, quando os pais não possuem um repertório de habilidades sociais adequadas, o desenvolvimento de habilidades sociais nas crianças pode ser prejudicado. Neste caso, a convivência familiar pode ser danosa, bem como as crianças podem sofrer transtornos associados a problemas internalizantes (depressão, ansiedade) ou externalizantes (agressividade, comportamento antissocial).

Em vários países, como Canadá e Estados Unidos, muitas clínicas investem no que é chamado de "Treinamento de Pais". Algumas delas incluem recursos para desenvolver treinamento de habilidades sociais educativas, aquelas que têm objetivo de promover intencionalmente a aprendizagem de comportamentos sociais adequados. No Brasil, vários pesquisadores vêm atuando com treinamento de pais associados ou não ao atendimento terapêutico da criança e da família e indicam que o processo seria mais efetivo se contasse com a inserção da família para manutenção e generalização dos efeitos do processo terapêutico (Del Prette \& Del Prette, 2005).

Pinheiro, Haase, Del Prette, Amarante e Del Prette (2006) relatam que o treinamento de pais tem sido aplicado para tratamento de problemas de comportamento como birras, agressões, desobediência excessiva. Os autores citaram pesquisadores que adaptaram um programa de treinamento para pais, com o objetivo de estimular a interação social em uma criança com epilepsia. A ideia foi introduzir um modelo triádico (terapeuta-pai-filho), no qual os pais aprendem 
conceitos teóricos com os terapeutas e, com base nessas aprendizagens, atuam como mediadores para manejar os comportamentos de seus filhos. Em seguida, Pinheiro et al. (2006) relatam os resultados que obtiveram por meio de um programa de Treinamento de Habilidades Sociais para pais de crianças com problemas de comportamento.

O programa ocorreu durante 11 semanas consecutivas, com a participação de 32 mães e dois pais. Durante a intervenção abordaram princípios da análise do comportamento para a prática disciplinar não coercitiva e modelos de habilidades sociais educativas para pais. Nesse sentido, os pais foram incentivados a: a) prestar atenção no bom comportamento do filho e elogiá-lo quando adequado; b) promover e monitorar a brincadeira independente da criança; dar instruções de maneira adequada; c) fazer leitura do ambiente social; d) desenvolver habilidades empáticas com as crianças, que incluem prestar atenção aos sinais que a criança emite quando está vivenciando algum problema, e) ouvir de maneira atenta o que a criança tem a dizer, colocar-se no lugar da criança, expressar verbalmente compreensão e apoio, entre outros. Adicionalmente, foi solicitado aos pais que realizassem tarefas semanais de observar o comportamento do filho, estabelecer condições de aprendizagem e desempenho de comportamentos desejáveis (empatia, seguimento de instruções, independência etc.), expressão afetiva entre outros. Os resultados indicaram significativa diminuição de frequência e severidade de comportamentos inoportunos ou indisciplinados, conforme a avaliação dos pais. A análise dos resultados qualitativos e quantitativos indicou que o programa foi bem sucedido, pois foram observadas melhoras significativas nos comportamentos inadequados das crianças e mudanças consideráveis nas interações intrafamiliares. Os autores concluíram que 0 enfoque de habilidades sociais educativas para pais pode contribuir positivamente para 0 desenvolvimento de práticas disciplinares não coercitivas.

\section{Discussão}

Esta revisão da literatura se justifica pela lacuna encontrada em estudos de periódicos brasileiros na área de fisioterapia, que não explicitam estratégias viáveis e eficazes para a promoção do envolvimento familiar na reabilitação de crianças. Assim, a proposta deste estudo foi buscar e apresentar estratégias utilizadas em outras disciplinas, com o objetivo de obter e qualificar a participação dos pais, fazendo articulação com contextos de reabilitação em fisioterapia com crianças, sob uma perspectiva de contribuição interdisciplinar. Educação Infantil, Educação Especial e Educação Musical têm em comum com a área de fisioterapia neuropediátrica: a 
importância da corresponsabilização e compartilhamento das metas; comprometimento do profissional e da família com o processo; necessidade de compartilhamento de informações; caráter duradouro das intervenções; necessidade de seguimento das instruções dos profissionais no contexto doméstico; sensibilidade dos profissionais para entender o contexto familiar, valorizando os pontos fortes da família; responsabilidade do profissional em promover o envolvimento familiar. Para contribuir com estas metas, o modelo de intervenção Treinamento de Pais com enfoque em Habilidades Sociais educativas pode ser um recurso importante.

Ainda que sejam necessários estudos empíricos que afiram a pertinência das estratégias encontradas nas áreas pesquisadas para a realidade do contexto de reabilitação em fisioterapia com crianças, discutem-se, a seguir possíveis entrelaçamentos com a área de fisioterapia.

Conforme ocorre nas áreas de Educação Infantil, poderia ser pertinente ao contexto de fisioterapia na reabilitação de crianças, recomendações explícitas para incluir a família nas intervenções e a encorajar a colaboração parental de forma sistemática, compartilhamento de informações sobre o desenvolvimento da criança, reuniões periódicas e conversas em espaços para pais, avaliação do programa de reabilitação em conjunto com a família, explicitadas nos documentos oficiais na esfera da Educação (MEC, 2009; Campos \& Rosemberg, 2009). A própria existência de documentos oficiais explicitando políticas e estratégias poderia impulsionar a prática profissional bem como o campo de estudo.

Considerando as estratégias apontadas por Campos e Rosemberg (2009) em relação à educação, parecem pertinentes se aplicadas à reabilitação de crianças com transtornos neurológicos, alterando o contexto da educação para a reabilitação: manter o diálogo aberto e contínuo com os pais; estímulo aos pais para participação ativa; orientação sobre a importância das brincadeiras (potencialmente reabilitadoras) para o desenvolvimento infantil; oferecimento de informações sobre o desenvolvimento das crianças; existência de espaços acolhedores para receber e conversar com as famílias.

Assim como existe o documento "Indicadores de Qualidade na Educação Infantil" (MEC, 2009) seria desejável que o mesmo ocorresse na esfera de reabilitação: um documento oficial com o objetivo de auxiliar as equipes, famílias e comunidade a participar de processos de autoavaliação da qualidade do atendimento, por exemplo. E entre os indicadores de qualidade constar, assim como no referido documento da área de educação infantil, a expressão da garantia do direito das famílias de acompanhar o tratamento e a evolução das crianças; atualização periódica do plano de tratamento com a participação dos profissionais e famílias; existência de espaços especialmente planejados para recepção e acolhimento dos 
familiares; reuniões com os familiares pelo menos três vezes por ano para apresentar planejamentos, discutir e avaliar o curso da reabilitação.

Indicadores de qualidade na reabilitação também poderiam ser descritos e avaliados por meio de um instrumento, conforme ocorre, no âmbito da educação infantil, com a escala ITERS-R (Harms et al., 2003). A escala se refere à conscientização da família sobre a filosofia do Programa, ao manual para pais, à descrição de atividades, às reuniões de orientação aos pais, ao compartilhamento de informações. Estas estratégias poderiam ser aplicadas no contexto da fisioterapia e poderiam ser consideradas uma operacionalização das grandes classes de comportamento referidas por Sari e Marcon (2008): esclarecer, incentivar, conscientizar pais sobre o tratamento fisioterapêutico de crianças.

Em relação às estratégias para aumentar a participação dos pais no acompanhamento do tratamento dos seus filhos, descritos na sessão de Educação Especial, considera-se a possibilidade de seguimento destas práticas em fisioterapia, principalmente porque parte das crianças atendidas na reabilitação fisioterapêutica infantil são crianças com necessidades especiais, como as crianças participantes nos estudos de Silva e Daltrário (2008), Vasconcelos et al.,(2009), Gração e Santos (2008). Assim como as áreas anteriormente descritas, as estratégias desta área incluíram a tomada de decisões sobre a intervenção com a criança em conjunto com a família, avaliação conjunta do processo terapêutico (Araújo, 2004), estratégias importantes para a área de fisioterapia. Além dessas possibilidades, outras que podem ser testadas são: a existência de um grupo de apoio para os cuidadores principais da criança; possibilidade de o fisioterapeuta, por meio de ação conjunta com o psicólogo e assistente social, conhecer as concepções dos pais, suas atitudes e valores para entender as dificuldades e barreiras que os cuidadores podem ter para realizar as atividades solicitadas ao longo da semana; identificar os limites da condição socioeconômica para recomendar o uso de equipamentos e serviços dentro do alcance dos cuidadores; criar e aproveitar oportunidades para desenvolver 0 respeito e confiança mútuos que é outro requisito para que os cuidadores resolvam fazer $o$ investimento de tempo, esforço e dinheiro que serão necessários para o sucesso do tratamento.

Outras indagações derivadas da observação da literatura na área de Educação Especial pertinentes ao fisioterapeuta rotineiramente: a intervenção envolve o maior número de familiares possível? Os pais estão empoderados para a reabilitação dos filhos? Têm ocorrido condições para esse empoderamento? Os pais compreendem os serviços, podem participar das decisões, adaptação dos procedimentos e liberdade para aceitar ou não objetivos 
estabelecidos? Antes, os profissionais oferecem condições para ocorrer tal empoderamento?

Além das estratégias da área de Educação Infantil e Educação Especial, retomam-se ideias na área de Educação Musical infantil. Como na Educação Musical pela abordagem Suzuki (Barber, 1991), propõe-se que seria vantajoso que na fisioterapia o pai fosse incentivado a participar das sessões do filho, tomar notas e tirar dúvidas para realizar atividades recomendadas pelo terapeuta em casa durante a semana; manter atitude positiva, fazer com que a criança execute os exercícios repetidamente, fazer perguntas ao fisioterapeuta. Estimular que os pais executem os exercícios que são exigidos para a criança ou realizados nela, para que se deem conta dos músculos, nervos, membros envolvidos, a possível dor associada e intensidade, para que sejam mais efetivos para conduzir a reabilitação no ambiente doméstico.

Seria importante que, durante o tratamento de fisioterapia, fossem oferecidas ideias de jogos e atividades para os pais realizarem com as crianças em casa visando à reabilitação, um leque de atividades como propõe Sprunger (2005), em relação à música. Essas práticas poderiam ser as necessárias para o alcance do objetivo de contar com a participação dos cuidadores no tratamento de fisioterapia dos filhos, uma vez que, segundo Lima (2006), a continuidade e manutenção do alinhamento biomecânico no domicílio depende da sua participação no tratamento fisioterapêutico.

Como descrito na área de Habilidades Sociais (Del Prette \& Del Prette, 2005), poderia ser importante, para reabilitação fisioterapêutica, ensinar e incentivar os pais a terem habilidades sociais adequadas para melhores resultados na relação interpessoal que permeia o processo de reabilitação. Como estratégia, descrita por Pinheiro et al. (2006), está a intervenção com pais, no modelo de Treinamento de Pais, muito utilizado na Psicologia, mas neste caso enfocando as habilidades sociais educativas.

Como no estudo de Pinheiro et al. (2006), o conteúdo do curso enfocando a fisioterapia poderia incentivar os pais a prestar atenção no bom comportamento do filho relacionado à reabilitação e elogiá-lo quando adequado - por exemplo, quando o filho aceitar usar órtese, quando permanecer em posições adequadas para o alinhamento biomecânico, quando o terapeuta relatar que a criança teve comportamento colaborativo na sessão de fisioterapia. Por outro lado, ensinar os pais a extinguirem comportamentos inadequados como birras, por exemplo. A capacitação dos pais poderia também ensinar a promover e monitorar a brincadeira independente da criança, já que a independência é um dos objetivos do tratamento de fisioterapia; além disso, dar instruções de maneira adequada; oferecer instruções objetivas, passo a passo; aguardar o desempenho da criança; decompor comportamentos complexos em unidades mais 
simples. Por fim, incentivar, por meio de conteúdo teórico e oportunidade prática, os pais a desenvolverem habilidades empáticas com as crianças. Estas habilidades incluem prestar atenção aos sinais que a criança emite quando está vivenciando algum problema (por exemplo, realizando um exercício importante para o tratamento, porém, desconfortável ou doloroso, como alongamentos), ouvir de maneira atenta o que a criança tem a dizer, colocando-se no lugar dela, e expressar verbalmente compreensão e apoio.

O estudo supracitado indica que, por meio da mediação profissional, os pais podem adquirir repertório importante, que poderia ser utilizado no contexto da reabilitação das crianças com disfunções neurológicas assim como no cotidiano da criança, promovendo relações mais satisfatórias e potencializando resultados mais efetivos. A efetividade das intervenções em Habilidades Sociais tem sido descrita em diversos estudos, como práticas baseadas em evidências (Del Prette \& Del Prette, 2011).

Em suma, observa-se que nas diversas áreas do conhecimento tratadas nesse trabalho são explicitadas estratégias que potencialmente poderiam contribuir para a prática da fisioterapia neuropediátrica para melhor promover o envolvimento parental no tratamento dos filhos, uma vez que estes mecanismos não estão claros ou facilmente acessíveis na literatura da área. Não se espera que essas estratégias sejam planejadas e utilizadas solitariamente pelo fisioterapeuta no processo de reabilitação, haja vista que tais conteúdos não fazem parte dos currículos do curso de Fisioterapia, mas que o trabalho e a produção de conhecimento sob a perspectiva interdisciplinar venha preencher essa lacuna. Os recursos e estratégias pontuadas nesse estudo podem ser importantes como ponto de partida para que os profissionais de Saúde - fisioterapeutas, psicólogos, assistentes sociais, as instituições de reabilitação e mesmo os pais busquem por mecanismos que promovam a participação da família nos contextos de intervenção fisioterapêutica visando maior efetividade na reabilitação de crianças. Estudos dessa natureza podem ser uma resposta à Lima (2006) quando trata da necessidade de estudos e práticas interdisciplinares em saúde.

Por fim, aponta-se para a necessidade de estudos empíricos junto aos fisioterapeutas e em contextos de reabilitação fisioterapêutica para aferir a pertinência e a importância dessas estratégias apresentadas para a promoção do envolvimento parental na reabilitação de crianças.

\section{Referências}

Amorim, R. C. A., Laurentino, G. E. C., Barros, K. M. F. T., Ferreira, A. L. P. R., Moura Filho, A. G., \& Raposo, M. C. F. (2009). Programa 
de saúde da família: proposta para identificação de fatores de risco para o desenvolvimento neuropsicomotor. Brazilian Journal of Physical Therapy, 13(6), 506-513.

Araújo, E. A. C. (2004). Parceria Família-Profissional em Educação Especial: Promovendo habilidades de comunicação efetiva. In E. G. Mendes, M. A. Almeida, \& L. C. A. Williams (Orgs.), Temas em Educação Especial: avanços recentes. (pp. 175-178). São Carlos: EDUFSCar.

Barber, B. (1991). Traditional \& Suzuki Teaching: a comparison. Suzuki Association of the Americas. <https://suzukiassociation.org/news/traditional-suzuki-teachingcomparison/>.

Campos, M. M., \& Rosemberg, F. (2009). Critérios para um atendimento em creches que respeite os direitos fundamentais das crianças. Brasília: MEC, SEB. Recuperado em 06 maio, 2010, de <http://portal.mec.gov.br/dmdocuments/direitosfundamentais. pdf> Campos, M. M., Esposito, Y. L., Bhering, E., Gimenes, N., \& Abuchaim, B. (2011). A qualidade da educação infantil: um estudo em seis capitais brasileiras. Cadernos de Pesquisa, 41(142), 20-54.

Cruz, C. M. V., Ide, M. R., Tanaka, C., \& Caromano, F. A. (2008). Elaboração e validação de manual de massagem para bebês. Fisioterapia em Movimento, 21(4), 19-26.

Del Prette, A., \& Del Prette, Z. A. P. (Orgs.). (2011). Habilidades sociais: intervenções efetivas em grupo. São Paulo: Casa do Psicólogo.

Del Prette, A., \& Del Prette, Z. A. P. (2005). Psicologia das habilidades sociais: Terapia, educação e trabalho. Petrópolis: Vozes.

Dessen, M. A., \& Pereira-Silva, N. L. (2004). A família e os programas de intervenção: tendências atuais. In E. G. Mendes, M. A. Almeida, L., \& A. C. Williams (Orgs.), Temas em educação especial (pp. 179187). São Carlos: Edufscar.

Dunst, C. J., \& Dempsey, I. (2007). Family-professional partnership and parenting Competence, Confidence, and enjoyment. International Journal of Disability, Development and Education, 54(3), 305-318, doi: 10.1080/10349120701488772

Foltz, L.C., Degangi, G., \& Lewis, D. (2007). Fisioterapia, terapia ocupacional e terapia da fala e linguagem. In E. Generalis (Org.), Crianças com paralisia cerebral: guia para pais e educadores (pp. 150-182). Porto Alegre: ArtMed.

Gração, D. C., \& Santos, M.G.M. (2008). A percepção materna sobre a paralisia cerebral no cenário da orientação familiar. Fisioterapia em Movimento, 21(2), 107-113.

Harms, T., Cryer, D. R., \& Clifford, R. M. (2003). Infant/Toddler Environment Rating Scale- Revised Edition. New York: Teachers College Press. 
Koren, P. E., De Chillo, N., \& Friesen, B. (1992). Measuring empowerment in families whose children have emotional disabilities: a brief questionnaire. Rehabilitation Psychology, 37(4), 305-321.

Lima, R. A. B. C. (2006). Envolvimento materno no tratamento fisioterapêutico de crianças com deficiência: compreendendo dificuldades e facilitadores. Dissertação de Mestrado, Faculdade de medicina, Universidade Federal de Minas Gerais, Belo horizonte. Recuperado em 12 outubro, 2010, de <http://ftp.medicina.ufmg.br/cpg/programas/saude_crianca/teses_di ssert/2006_mestrado_regina_lima.pdf> Martinez, C. M. S., Joaquim, R. H. V. T., Oliveira, E. B., \& Santos, I. C. (2007). Suporte informacional como elemento para orientação de pais de pré-termo: um guia para o serviço de acompanhamento do desenvolvimento no primeiro ano de vida, Brazilian Journal of Physical Therapy, 11(1), 73-81.

Meireles, B. H. S., \& Erdmann, A. L. (1999). A questão das disciplinas e da interdisciplinaridade como processo educativo na área da saúde. Texto Contexto Enfermagem, 8(1), 149- 65.

Menegasso, L. R., \& Barham, E. J. (2011). Estratégias usadas para promoção do envolvimento parental em estudos de fisioterapia brasileiros: um estudo de revisão. Arquivos de Ciências da Saúde, 18(3), 119- 129.

Menegasso-Gennaro, L. R., \& Gil, M. S. C. A. (2012). Análise teórica de itens de uma escala americana para avaliação do atendimento em creches inclusivas brasileiras. Revista Educação Especial, 25(44), 531-544.

Ministério da educação. Secretaria da educação básica. (2009). Indicadores da Qualidade na Educação Infantil. Recuperado em 06 maio, 2010, de $<$ http://portal.mec.gov.br/dmdocuments/indic_qualit_educ_infantil.p df>

Pinheiro, M. I. S., Haase, V. G., Del Prette, A., Amarante, C. L. D., \& Del Prette, Z. A. P. (2006). Treinamento de Habilidades Sociais e Educativas para Pais de Crianças com Problemas de Comportamento. Psicologia: Reflexão e Crítica, 19(3), 407-414.

Prado, M. S. S., Magalhães, L. C., \& Wilson, B. N. (2009). Crosscultural adaptation of the developmental coordination disorder questionnaire for Brazilian children, Brazilian Journal of Physical Therapy, 13(3), 236-243.

Resolução CNE/CSE no 4. (2002, 19 de fevereiro). Recuperado em 10 maio, 2010, de <http://portal.mec.gov.br/cne/arquivos/pdf/CES042002.pdf>

Santos, D. C. C., Tolocka R. E., Carvalho, J., Heringer, L. R. C., Almeida C. M., \& Miquelote, A. F. (2009). Desempenho motor grosso e sua associação com fatores neonatais, familiares e de exposição à 
creche em crianças até três anos de idade. Brazilian Journal of Physical Therapy, 13(2), 173-179.

Sari, F. L., \& Marcon, S. S. (2008). Participação da família no trabalho fisioterapêutico em crianças com paralisia cerebral. Revista brasileira de crescimento e desenvolvimento humano, 18(3), 229239. Recuperado em 06 maio, 2010, de <http://www.revistasusp.sibi.usp.br/pdf/rbcdh/v18n3/03.pdf>.

Silva, M. A., \& Pfeifer, L. I. (2007). Reabilitação pulmonar de crianças com fibrose cística do estado do Pará. Fisioterapia em Movimento, 20(4), 73-81.

Silva, M. S., \& Daltrário, S. M. B. (2008). Paralisa cerebral: desempenho funcional após treinamento da marcha em esteira. Fisioterapia em Movimento, 21(3), 109-115. Recuperado em 06 maio, 2010, $<$ www2. pucpr. br/reol/index. php/RFM?dd1 $=2072 \& d d 99=\mathrm{pdf}>$

Silva, N. C. B. (2007) Contexto familiar de crianças com Síndrome de Down: interação e envolvimento paterno e materno. Dissertação de Mestrado. Universidade Federal de São Carlos. Recuperado em 12 outubro, 2010 , <http://200.136.241.56/htdocs/tedeSimplificado/tde_busca/arquivo. php? codArquivo $=1422>$

Silva, P. L., Santos, D. C. C., \& Gonçalves, V. M. G. (2006). Influência de práticas maternas no desenvolvimento motor de lactentes do 6 o ao 12 o meses de vida. Revista brasileira de fisioterapia, 10(2), 225231. <http://dx.doi.org/10.1590/S1413-35552006000200014>

Silva, N. C. B. (2010). Contexto familiar de crianças com Síndrome de Down: interação e envolvimento materno e paterno. Dissertação de Mestrado em Educação Especial. Universidade Federal de São Carlos, São Carlos. Recuperado em 06 outubro, 2010, de <http://200.136.241.56/htdocs/tedeSimplificado/tde_busca/arquivo. php? codArquivo $=1422>$

Souza, T. N., \& Campos-de-Carvalho, M. I. (2005). Qualidade de ambientes de creche: uma escala de avaliação. Psicologia em Estudo, 10(1), 87-96.

Sprunger, E. (2005). Helping parents practice ideas for making it easier. Yes Publishing: Saint Louis.

Vasconcelos, R. L. M., Moura, T. L., Campos, T. F., Lindquist, A. R. R., \& Guerra, R. O. (2009). Avaliação do desempenho funcional de crianças com paralisia cerebral de acordo com níveis de comprometimento motor. Brazilian Journal of Physical Therapy, 13(5), 390-397.

Williams, L. C. A., \& Aiello, A. L. R. (2004). Empoderamento de famílias: o que vem a ser e como medir? In E. G. Mendes, M. A. Almeida, \& L. A. C. Williams (Orgs.), Temas em educação especial: avanços recentes (pp. 197202). São Carlos: Edufscar. 


\section{Endereço para correspondência}

\section{Lisandrea Rodrigues Menegasso Gennaro}

Unidade Saúde-Escola, Universidade Federal de São Carlos

Rodovia Washington Luís, km 235 - SP-310, CEP 13565-905, São Carlos - SP, Brasil

Endereço eletrônico: lisandrea@ufscar.br

\section{Elizabeth J oan Barham}

Departamento de Psicologia, Universidade Federal de São Carlos

Rodovia Washington Luís, km 235 - SP-310, CEP 13565-905, São Carlos - SP, Brasil

Endereço eletrônico: lisa@ufscar.br

Recebido em: 10/05/2012

Reformulado em: 01/10/2013

Aceito para publicação em: 07/10/2013

Acompanhamento do processo editorial: Deise Maria Leal Fernandes Mendes

\section{Notas}

* Psicóloga na Universidade Federal de São Carlos. Doutoranda no Programa de Pós- Graduação em Psicologia da Universidade Federal de São Carlos

** Docente do Departamento de Psicologia e Programa de Pós- Graduação em Psicologia na Universidade Federal de São Carlos. 